neutron capture therapy because of the theoretically insufficient neutron intensity, the future of such a therapy for surgically inoperable brain tumors is promising, on conditions, that specifically designed nuclear reactors are built, and that the patients to be treated were not previously treated by conventional radiotherapy.

The indications are, 1) infiltrative tumors, like gliomas, 2) inoperable tumors, like pontine gliomas or thalamus tumors, 3) incompletely excisable tumors, like parasagittal meningiomas, 4) intramedullary tumors, and 5) inevitably complicating tumors, like some acoustic neurinomas.

\title{
E-6. Studies on the Uptake of Anticancer Drug into Brain Tumor Tissue
}

\author{
Kazuo Takeuchi, Masayuki Atsuchi, Takeshi Masui, \\ Toshi Sueyoshi, Mitsuhiro Hara and Ryuji Ogrwara \\ Department of Neurosurgery, Toranomon Hospital, Tokyo
}

In case of the systemic chemotherapy of brain tumor, uptake of sufficiently high concentration of the chemotherapeutic agent into the tumor tissue must be necessary, before the toxic side-effects of the drug will appear. For such uptake of the drug, its affinity to the tumor tissue or a breakdown of the blood-brain barrier are thought to be beneficial.

Bleomycin, an antitumor antibiotic specially accumulating into the skin, lung etc., has been used in 120 cases of brain tumor by the authors. Because it gives excellent results that had never been attained with the chemotherapeutic agents heretofore introduced, its concentration in the tumor tissue was measured by bioassay. No uptake of Bleomycin level either into the tumor tissue or brain tissue could be found in all of the 12 cases of meningioma and meningosarcoma. It was, howver, found at a level of $0.3 \mathrm{r} / \mathrm{ml}$ in the encysted fluid and $0.9 \mathrm{r} / \mathrm{mg}$ in the tumor tissue in only one case of astrocytoma, grade 3 out of 10 cases of glioma. In this particular case, nearly the same concentration was detected in encysted fluid examined one year ago. Bleomycin was also detected in the cystic fluid of each one case of granuloma and congenital subarachnoidal cyst.

According to the study on the protein fraction of encysted fluid of 10 cases of brain tumor including 6 cases of glioma, cyst formation of the tumor is thought to be caused by diffusion of plasma from the capillaries of the tumor by a manifestation of the blood-brain barrier phenomenon. Then, detection of Bleomycin in the cyst fluid of the brain tumor can be explained by its penetration of the blood-brain barrier.

Result of the comparative studies on brain scintiscanning, concentration of chemotherapeutic agent and protein fraction of the cyst fluid on 12 cases of cystic brain tumor is not constant. Moreover, positive brain scanning can be obtained more frequently in cases of malignant brain tumors such as glioblastoma and metasta- 
tic tumors. Whereas chemotherapy of brain tumor is less effective against such the malignant tumors. Therefore, mearly breakdown of blood-brain barrier in the tumor tissue is not so worthy mentioning for more successful chemotheraphy of brain tumors.

\title{
E-7. Intra-Tumor Irrigation of the Anticancer Agents for the Treatment of Brain Tumors
}

\author{
Minoru Aoyagi, M. D. \\ Department of Neurosurgery, Kurashiki Central Hospital, Kurashiki \\ (Department of Neurosurgery, Kyoto University Medical School, Kyoto) \\ Tomio Ohta, M. D. \\ Department of Neurosurgery, Osaka City University Medical School, Osaka \\ Hajime Handa, M. D. \\ Department of Neurosurgery, Kyoto University Medical School, Kyoto
}

Chemotherapy has been problematical of its practical efficiency in the treatment of malignant neoplasma of the central nervous system. The results so far, however, have been discouraging.

Chemotherapy for the brain tumor is to be ruled on the basis of Ehrlich's "Thearapia sterilisans magna". On the other hand, it is necessary and practically important for the brain to be strictly protected from cytocidal effect of the anticancer against the tumor. There have been no monospecific antitumor agents which are conventionally administered via the cerebrovascular bed. The intravenous administration of the agents resulted in their inherent noxious side-effects overcoming the essential antitumor effects widely in the systemic category. The technique of application of the antitumor agents into the regional artery could not avoid their systemic leakage and concomitant collateral brain edema or swelling which should always restrict the total effect of the agents. Neurosurgery followed by the local application of the antitumor agents was for the first time reported by Heppner and Diemath (1962), and thereafter Ringkj $\phi$ b reported 40 cases of the brain tumors treated by local application of cytostatic drugs (1968). The authors have already reported one case of metastatic brain tumor which was treated successfully by only intratumor irrigation of the anticancer agent without major craniotomy (1969).

The authors are now presenting the technique of intratumor irrigation of the anticancer agents for the treatment of brain tumor and eight clinical cases treated by this technique. The technique consists of five procedures $((1)$ catheterization, (2) fistulation, (3) irrigation and cavitation, (4) neutralization, and (5) decatheterization), and the tumor is to be selectively irrigated by the mixture of the high concentration of antitumor agents and lysosome labilyzer for the purpose of accentuation of intracellular uptake of cytocidal drugs and the purpose of liquefaction of the 